\title{
Pressure-induced oligomerization of alanine at room temperature
}

\author{
H. KAGi ${ }^{* 1}$, S. OdA ${ }^{1}$, C. FUJimoto ${ }^{1}$, K. MimurA ${ }^{2}$
}

${ }^{1}$ Geochemical Research Center, Graduate School of Science, The University of Tokyo, Tokyo 113-0033, Japan

(kagi@eqchem.s.utokyo.ac.jp)

${ }^{2}$ Department of Earth and Planetary Science, Graduate Schoool of Environmental Studies, Nagoya University, Nagoya 464-8601, Japan

Amino acids are building blocks of proteins and the oligomerization of amino acids is the first step of the chemical evolution. We found pressure-induced oligomerization from alanine to alanylalanine and trialanine was reported at pressures from $5 \mathrm{GPa}$ to $11 \mathrm{GPa}$ at room temperauture (Fujimoto et al., 2015). Moreover, it was clarified that freeze concentration at high pressure enhances the oligomerization of alanine even on solid-free dilute aqueous solution of alanine. These findings may suggest that prebiotic oligopeptide formation at high pressure such as in the interior of icy planets. In the present study, we are going to report the maximum length of oligomers and the reaction mechanism of pressure-induced oligmerization of amino acids.

High-pressure experiments were conducted using a nontoroidal opposed anvil assembly with a sample volume of 10 $\mathrm{mm}^{3}$. Alanine powder was loaded in a metal encapsulating gasket and soaked with pure water. In this study, we prepared deffect-containing alanine sample by grinding alanine powder for 20 hours for investigating effects of lattice defect to oligomerization at high pressure. The recovered samples were analyzed using an LC-MSMS.

We have detected up to 12-mers of alanine oligomers from a sample recovered from $11 \mathrm{GPa}$. Diketopiperaznie (DKP) which is a ring dimer of alanine was below the detection limit. Formation of long-chain peptides predominates in the pressure-induced oligomerization of amino acids. By grinding alanine powder for 20 hours, formations of alanylalanine (dimer) and trialanine increased for approximately 3 times. The results suggest that pressureinduced oligomerization was notably enhanced by introduning lattice defects in alanine. In natural enviroments, amino acid molecules confined in a cage of minerals such as zeolite may result in high yield of peptide formation at high pressure. We propose a possobility of prebiotic peptide formation at high pressure such as in the interior of icy planets. 